\title{
Congenital Ostium Secundum Atrial Septal Defect with Mitral Valve Prolapse and Mitral Regurgitation - A Case Report and Review of Literature
}

\author{
MD. ASLAM HOSSAIN ${ }^{1}$, DHARMENDRA JOSHI ${ }^{1}$, MAYANK ACHARYA ${ }^{1}$, TAREQ MORSHED ${ }^{1}$, OMAR \\ SADEQUE KHAN ${ }^{1}$, SAMIR AZAM SUNNY ${ }^{1}$, MD. MUKHLESUR RAHMAN ${ }^{2}$ \\ ${ }^{1}$ Department of Cardiac Surgery, Bangabandhu Sheikh Mujib Medical University, Dhaka, Bangladesh, \\ ${ }^{2}$ Department of Cardiology, Bangabandhu Sheikh Mujib Medical University, Dhaka, Bangladesh,
}

Address for Correspondence: Dr. Md. Aslam Hossain, Associate Professor, Department of Cardiac Surgery, Bangabandhu Sheikh Mujib Medical University, Bangladesh.Email: draslamcts@gmail.com

\begin{abstract}
Mitral regurgitation (MR) associated with secundum Atrial Septal Defect (ASD) is not widely recognized but the association is not unusual. MR has been found in less than 10\% of adults with large ASD which is mainly caused by mitral valve prolapse (MVP). We are reporting a case of congenital ostium secundum ASD with MVP associated with MR, review the clinical relevance of this association, and discuss the related literature. Our patient has shown dramatic improvement in symptoms and signs after ASD closure with mitral valve replacement along with improvement in tricuspid regurgitation. More general awareness of this association will further help the surgeon for better management of the patient with this rather unusual combination of lesions.
\end{abstract}

Keywords: Atrial Septal Defect, Mitral Valve Prolapse, Mitral Regurgitation

Introduction:

An ostium secundum Atrial Septal Defect (ASD) is an abnormally large opening in the atrial septum at the site of the foramen ovale and the ostium secundum.Secundum ASD is not widely associated with Mitral regurgitation (MR) although the association is not unusual.Mitral valve (MV) prolapse which is caused by abnormal geometry of the left ventricle (LV) and MV apparatus secondary to right ventricular (RV) volume overload is the major cause of MRwhich is found in less than $10 \%$ of adults with large ASD. ${ }^{[1]}$ Researches have shown disease of mitral valve prolapse, congenital valve deformity, rheumatic valve, and specific pathophysiology complex as main causes of mitral regurgitation in patient with ASD. ${ }^{[2]}$

In this study, we report a case of congenital ostium secundum ASD with MVP attributing to MR, review the clinical relevance of this association, and discuss the related literature.

\section{Case Report:}

A 30-years-old, male, ex-smoker, nondiabetic, normotensive patient presented with history of shortness of breath on exertion along with palpitation for last 15 years. Shortness of breath has progressively aggravated lately for 2 months, associated with orthopnea and Paroxysmal Nocturnal Dyspnoea (PND). He had occasional palpitation with moderate exertion which isrelieved by taking rest. He gave history of productive cough with one episode of hemoptysis. He had generalized weakness of his body for last 2 months and occasional large joint pain from childhood along with mild fever which is subsided by medication. He had no history of chest pain, swelling of legs, syncope, loss of consciousness, hoarseness of voice or difficulty in swelling.

On physical examination, patient was ill looking, average body built, mildly anemic and had no jaundice, edema, cyanosis, clubbing or raised JVP. His height: $165 \mathrm{~cm}$, weight: $47 \mathrm{~kg}$, pulse: $84 \mathrm{bpm}$, regular, blood pressure: 100/60 $\mathrm{mmHg}$, respiratory rate: 12 breaths $/ \mathrm{min}$. Cardiovascular examination revealed epigastric pulsation along with right parasternal heave. The heart sounds were normal in all the areas except mitral area where the first sound was soft. There was wide and fixed splitting of second heart sound with soft flow murmur in left 2nd intercostal space. All other systemic examinations including respiratory and neurological system revealed normal findings. 

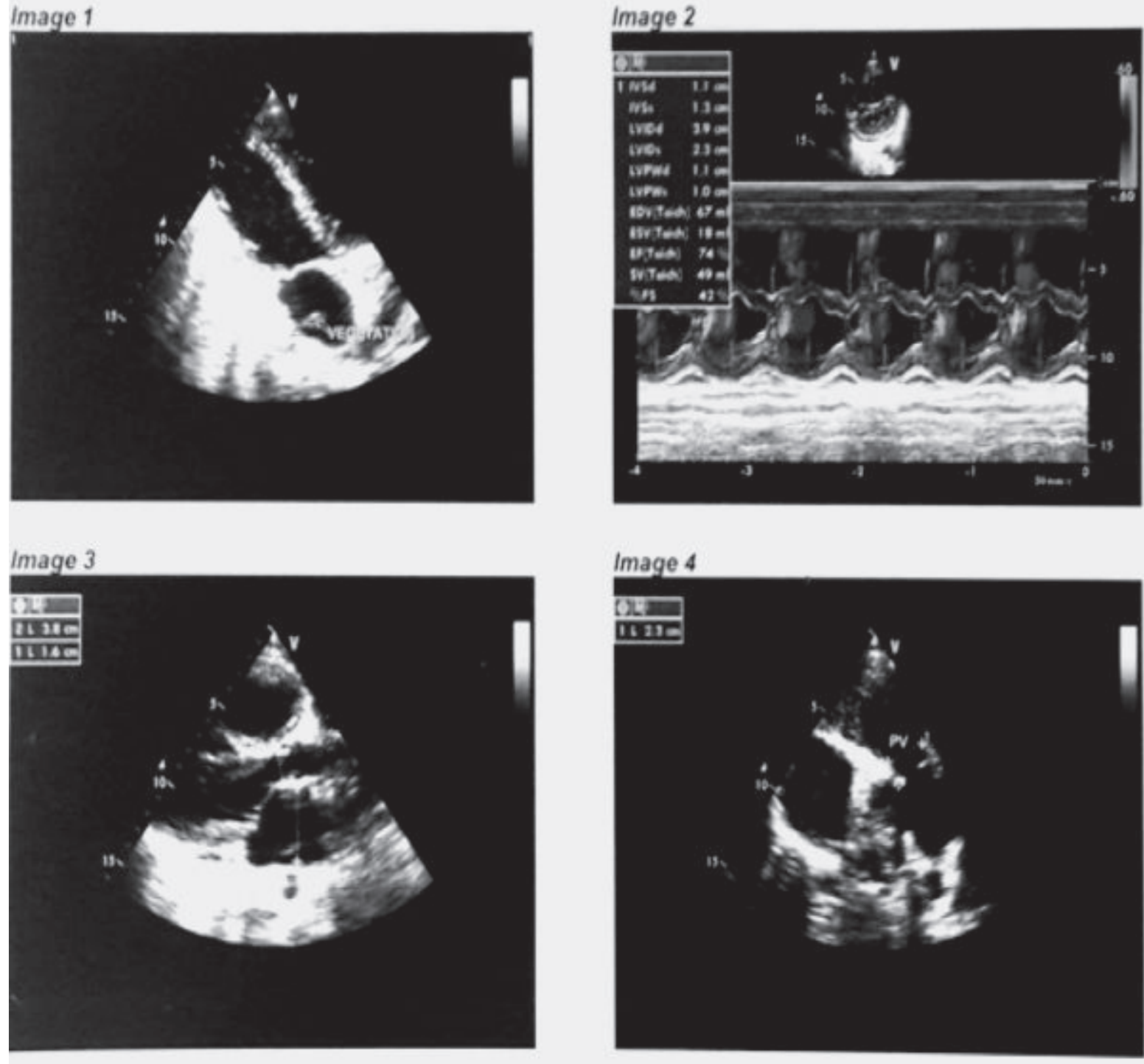

Image 4
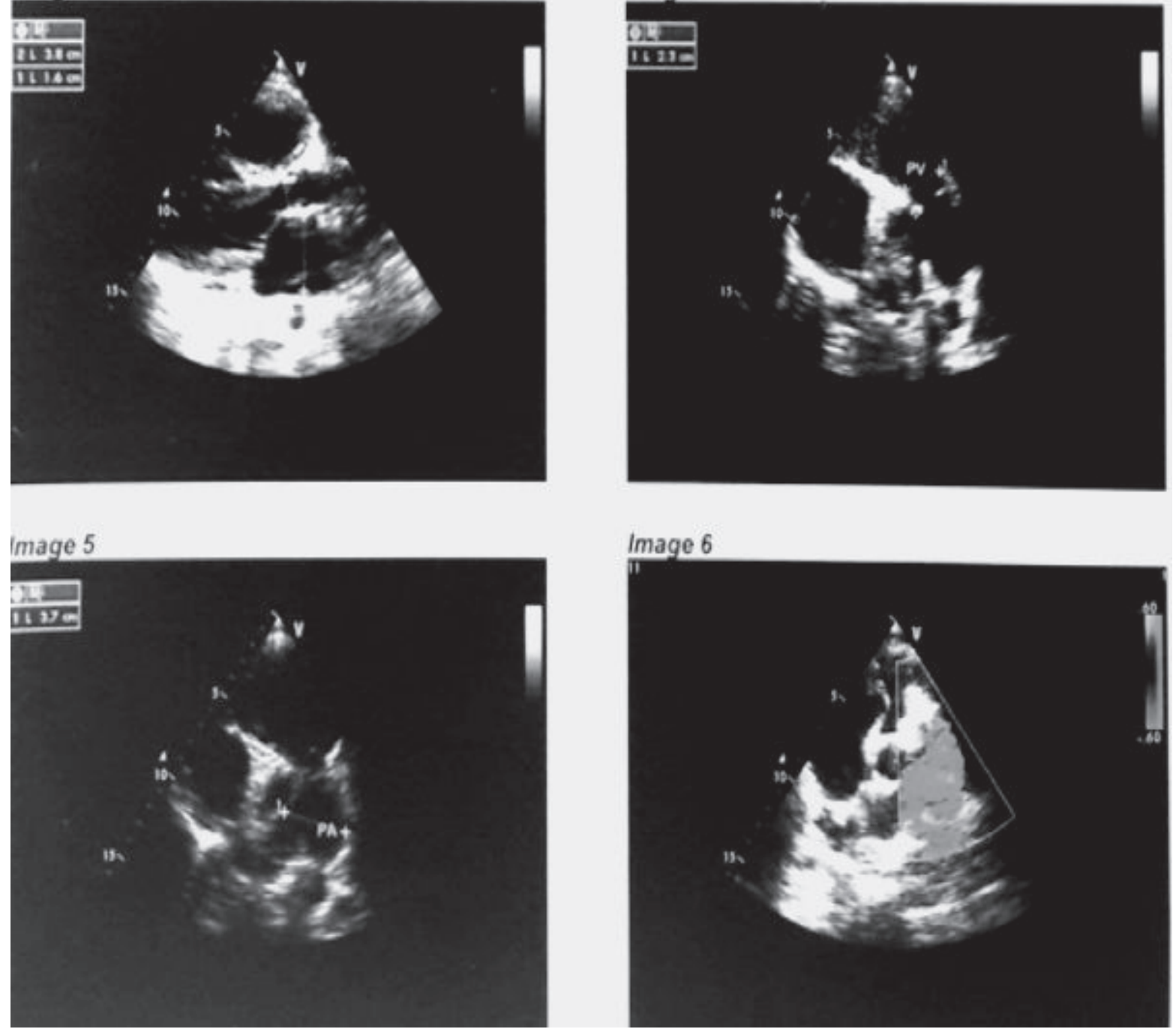

Fig.-1: Preoperative Echocardiography 

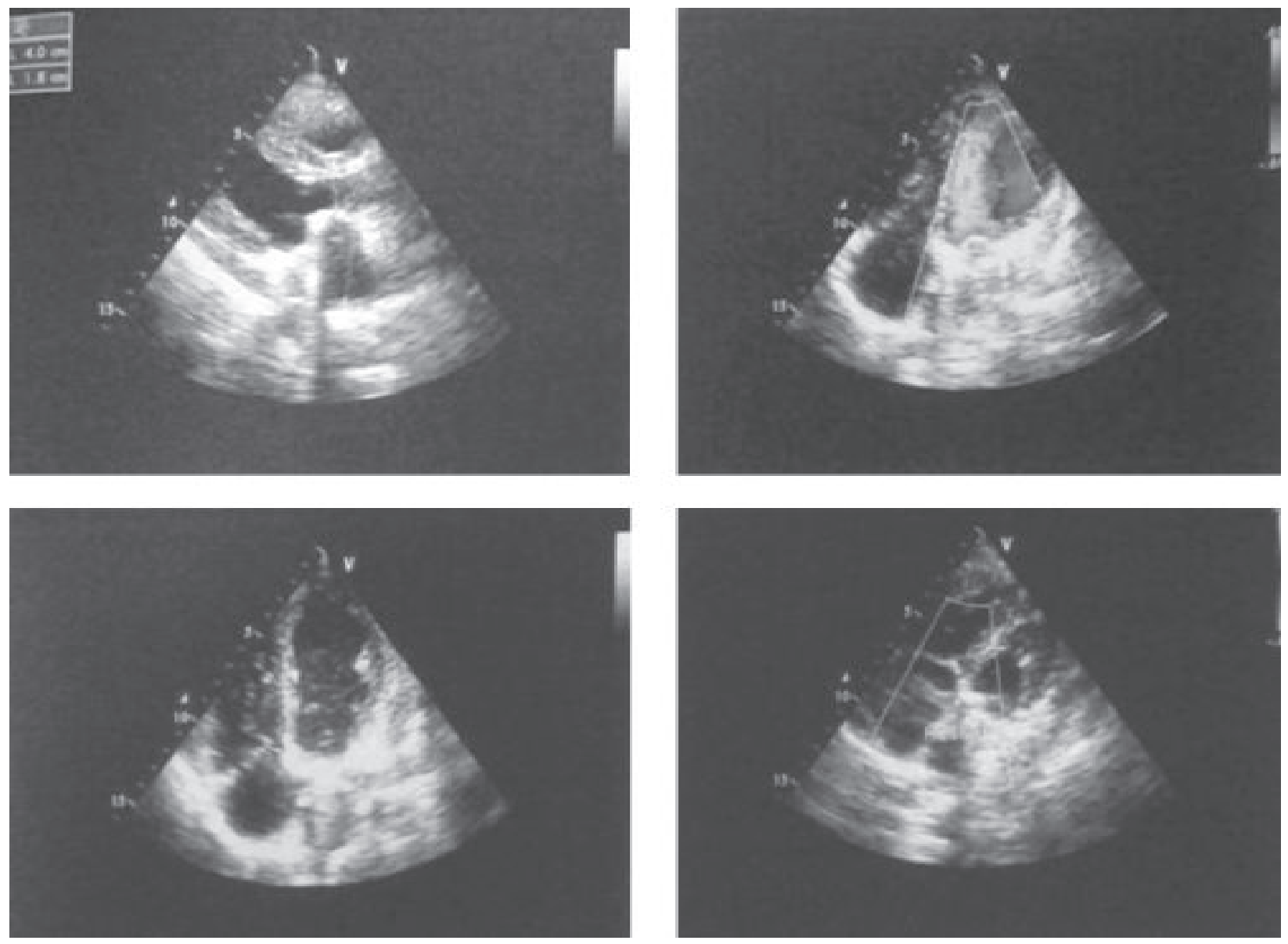

Fig.-2: Postoperative Echocardiography

Further laboratory evaluation showed, ESR $=43 \mathrm{~mm}$ in 1 st hour, serum albumin $=30 \mathrm{gm} / \mathrm{dL}$ (reference range 35 $50 \mathrm{~g} / \mathrm{L}$ ), CRP was initially $67.89 \mathrm{mg} / \mathrm{L}$ which was corrected to $7.23 \mathrm{mg} / \mathrm{L}$ before operation (reference range: $<6 \mathrm{mg}$ / L).Echocardiography (Fig: 1) showed ASD secundum $(25 \mathrm{~mm} X 25 \mathrm{~mm})$ with left to right shunt, moderate valvular $\mathrm{PS}(\mathrm{PPG}=58 \mathrm{mmHg}$ ) with mild PR, Mitral Valve Prolapse (AML) with moderately severe Mitral Regurgitation, dilated RA and RV with features of both volume and pressure overload, moderate $\mathrm{TR}(\mathrm{PPG}=73 \mathrm{mmHg})$, good $\mathrm{LV}$ and $\mathrm{RV}$ systolic function with $\mathrm{LVEF}=74 \%$. There were two vegetations at separate location: One vegetation was attached to the apical part of AML of MV on LV side and another vegetation was attached to the posterior wall of LA on the pathway of MR jet.

The patient was prepared for operation with furosemide, propranolol, prednisolone (for raised CRP level). After thoroughpre-operative preparation with proper counselling and informed written consent, he underwent ASD Closure with Mitral valve replacement on 24/04/
2017. ASD was directly closed with 4-0 prolene. Mitral valve was excised and replaced with $29 \mathrm{~mm}$ size metallic St. Judge mechanical valve in anti-anatomical position with continuous suture after evacuation of vegetations. Pulmonary valve was checked with 18 Hagar's dilator. Tricuspid valve was also checked with saline test and found good coaptation and minimal regurgitation. Total cross clamp time was 71 minutes and total cardiopulmonary bypass time was 122 minutes.

His post-operative period was uneventful. He wasextubated in ICU after 5 hours. Mediastinal drain tube and urinary catheter were removed on 2nd Post-Operative Day (POD). The patient was discharged on 9th POD on $03 / 05 / 2017$ with proper discharge instructions and follow up plan.

After one month of operation, the patient was clinically stable. Echocardiography (Fig: 2) showed normally functioning prosthetic mitral valve found in situ with PPG $=14.5 \mathrm{mmHg}$ and $\mathrm{MPG}=5.0 \mathrm{mmHg}$, Intra-atrial septum (IAS) found intact with residual mild valvular PS with 
mild PR, trivial TR (PPG $=26 \mathrm{mmHg}$ ), minimal localized pericardial effusion and good LV and RV systolic function. $\mathrm{LVEF}=62 \%$.

\section{Discussion:}

Congenital ostium secundum ASD associated with Mitral Valve Prolapse (MVP) leading toMR is rare occurrence. Association between ostium secundum ASDs and mitral valve prolapse is seen in about 10 to $20 \%$ of individuals. ${ }^{3}$

Mitral insufficiency is invariably linked with ostium primum ASD. However, like our case, it is also frequently seen in ostium secundum or venous sinus ASD. ${ }^{4}$ Many studies have found that dilatation of right ventricle caused by abnormal geometry of the left ventricle is the major reason for mitral valve changes. This incidence increases with age which can progress after operation done during adulthood. ${ }^{1}$

Most of the patients with MVP are asymptomatic. However, systolic clicks or mid to late systolic murmur during auscultation helps to diagnose MVP. ASD alter the clinical course of MR and MVP. Some patients with severe MR may be asymptomatic because the ASD may unload the left atrial volume making prompt diagnosis very difficult. ${ }^{[5]}$ However, the patients like our case, usually presents with dyspnea or palpitations and sometimes diagnosis is done on routine medical examination. Echocardiography confirmed the diagnosis in our case. Other studies also confirmed the use of echocardiography for diagnosis as standard technique. ${ }^{6}$

In patient with ASD, there is volumetric overload of the small annuli that leads to progressive dilatation of the right ventricle and further increases pulmonary pressure. Although such hemodynamic changes are well tolerated for decades but later it causes right ventricular dysfunction and cardiac insufficiency.Consequently, annular dilatation produces tricuspid valve insufficiencywhich further aggravate the sequences of ASD in adults. ${ }^{6}$
There are few literatures only which describes the association between ASD and tricuspid valve insufficiency than mitral valve insufficiency. The majority of aforementioned changes normalize when the ASD is closed before the age of 18 years.And echocardiography provides valuable confirmation of significant regression of tricuspid valve regurgitation in follow up of pre and post-operative cases. ${ }^{6}$ This coincides with our results that encourage surgery without delay.

\section{Conclusion:}

Mitral regurgitation (MR) associated with secundum ASD is not widely recognized but the association is not unusual. Patient may remain asymptomatic or present with variable symptoms. Echocardiography is the gold standard for the diagnosis in almost all the cases. The patient gets rid of the symptoms dramatically after surgical closure of congenital ASD and mitral valve replacement. So, surgical correction is recommended as soon as possible in adult cases of congenital ASD with MVP and MR.

\section{References}

1. Toyono, M., Pettersson, G.B., Matsumura, Y., Wada, N., Fukuda, S., Yamano, T.et al. Preoperative and postoperative mitral valve prolapse and regurgitation in adult patients with secundum atrial septal defect. Echocardiography 2008;25:1086-1093.

2. Waikittipong, S. Mitral regurgitation associated with secundum atrial septal defect. Thai J Surg 2010;31:120-124.

3. Kestelli, M. Mitral valve prolapse in atrial septal defect. Int J Cardiol 2001;1:1-4.

4. Oliver, J.M., Gallegoa, P., González, A.E,Benito, F., Sanz, E., Aroca, A. et al. Surgical closure of atrial septal defect before or after the age of 25 years. Comparison with the natural history of unoperated patients. Rev EspCardiol 2002;55(9):953-961.

5. Kang, J. and Das, B. Emergent presentation of decompensated mitral valve prolapse and atrial septal defect. West J Emerg Med. 2015;16(3):432-434.

6. Shaalan, A.M., Elwakeel, E.E, Elrakhawy, H.M, Alassal, M.A., and Gomaa, A. Improvement after surgical closure of secundum atrial septal defects in adults. J ClinExpCardiol 2017;8(1):493. 